\title{
Regions of Positive Vorticity in Steady Axisymmetric Flow Past a Viscous Spherical Drop
}

\author{
G. M. Moremedi ${ }^{1}$ and D. P. Mason ${ }^{2}$ \\ ${ }^{1}$ Department of Mathematical Sciences, University of South Africa, P.O. Box 392, Pretoria, South Africa \\ ${ }^{2}$ School of Computational and Applied Mathematics, University of Witwatersrand, Johannesburg, \\ Private Bag 3, Wits 2050, South Africa \\ Correspondence should be addressed to D. P. Mason; david.mason@wits.ac.za
}

Received 7 March 2013; Accepted 13 April 2013

Academic Editor: Fazal M. Mahomed

Copyright (c) 2013 G. M. Moremedi and D. P. Mason. This is an open access article distributed under the Creative Commons Attribution License, which permits unrestricted use, distribution, and reproduction in any medium, provided the original work is properly cited.

The vorticity exterior and interior to a viscous liquid drop in steady motion in an unbounded fluid is investigated. The perturbation solution to first order in the Reynolds number derived by Taylor and Acrivos (1964) is used. New analytical results are derived for the attached region of positive vorticity behind the drop and for the region of positive vorticity inside the drop.

\section{Introduction}

In this paper we will consider the steady axisymmetric flow of a viscous fluid past a liquid drop with constant interfacial tension. From numerical investigations [1-4] it is known that a detached wake consisting of a standing eddy and a region of positive vorticity exist outside the viscous drop if the Reynolds number, Re, and the ratio of the viscosity of the drop to the viscosity of the surrounding fluid, $\kappa$, are sufficiently large and that a region of positive vorticity exists inside the drop if either Re is sufficiently large or $\kappa$ is sufficiently small. In a recent paper [5] new analytical results for the detached wake and the streamlines inside and outside the liquid drop were derived using the singular perturbation solution for the stream function derived by Taylor and Acrivos [6]. In this paper we will derive corresponding analytical results for the equivorticity lines and for the regions of positive vorticity outside and inside the liquid drop.

The singular perturbation solution of Taylor and Acrivos [6] will again be used. This perturbation solution has two parameters, Re and $\kappa$. Although it was derived assuming that $\operatorname{Re}<1$, we will apply it to flows with $\operatorname{Re}>1$. There is evidence that the predictions of this solution are applicable for Re $>1$. For example, Wellek et al. [7] found that the Taylor and Acrivos perturbation solution predicted quite accurately the eccentricity of the deformed drop for Reynolds numbers up to $\operatorname{Re}=20$. Also as $\kappa \rightarrow \infty$, the solution of Taylor and Acrivos tends to the singular perturbation solution of Proudman and Pearson [8] for the stream function for slow viscous flow past a solid sphere. Van Dyke [9] found that the length of the attached wake behind a solid sphere calculated from the Proudman and Pearson perturbation solution to first order in the Reynolds number is in good agreement with experimental and numerical values for Reynolds number up to about $\mathrm{Re}=60$.

We will assume also that the interfacial tension is large so that the Weber number, We, is small, and therefore the deformation of the spherical drop is small.

An outline of the paper is as follows. In Section 2 the assumptions of the model are stated and the perturbation solution of Taylor and Acrivos for the stream functions outside and inside the liquid drop to first order in the Reynolds number Re is presented. In Section 3 the equivorticity lines and the attached region of positive vorticity exterior to the liquid drop are investigated. In Section 4 the equivorticity lines and the region of positive vorticity inside the liquid drop are considered. Finally the conclusions are summarised in Section 5. 


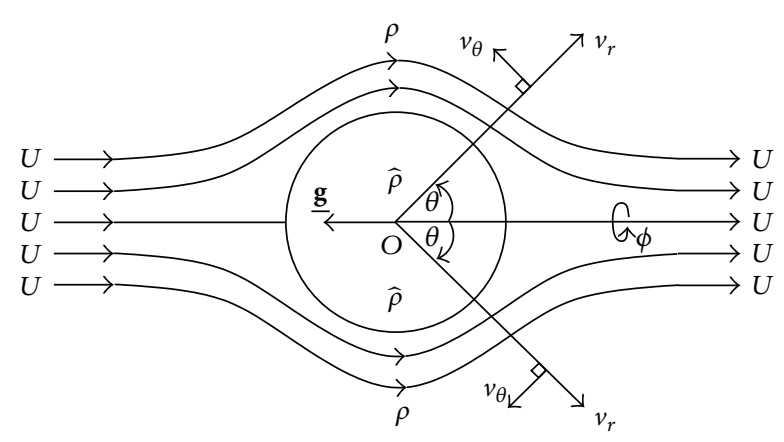

FIGURE 1: Spherical polar coordinate system $(r, \theta, \phi)$ for axisymmetric flow past a liquid drop. If $\hat{\rho}>\rho$, the gravity vector $\mathbf{g}$ points in the direction shown while if $\hat{\rho}<\rho, \mathbf{g}$ points in the opposite direction.

\section{Stokes Stream Functions}

In this section we will summarise the assumptions made in the derivation of the perturbation solution of Taylor and Acrivos [6] and state the Stokes stream functions inside and outside the liquid drop.

Consider the steady axisymmetric motion under gravity of a viscous liquid drop slightly deformed from the spherical shape in an unbounded quiescent fluid. The fluids inside and outside the liquid drop are incompressible and immiscible and the interfacial tension is assumed to be uniform. Fluid variables inside the liquid drop are denoted by a circumflex. The terminal velocity of the drop is $U$. The spherical polar coordinate system $(r, \theta, \phi)$ shown in Figure 1 with origin at the centre of mass of the drop is used. The fluid variables are dimensionless, and since the flow is axisymmetric, they are independent of $\phi$. The characteristic length is the radius, $a$, of the spherical drop with the same volume, and the characteristic velocity is the terminal velocity, $U$.

Since the fluids outside and inside the liquid drop are incompressible and the flow is axisymmetric with $v_{\phi}=0$ and $\widehat{v}_{\phi}=0$, Stokes stream functions, $\psi(r, \theta)$ and $\widehat{\psi}(r, \theta)$, can be introduced outside and inside the liquid drop. They are defined in terms of the velocity components parallel to the $r$ and $\theta$ coordinate lines by

$$
\begin{array}{ll}
v_{r}(r, \theta)=\frac{1}{r^{2} \sin \theta} \frac{\partial \psi}{\partial \theta}, & v_{\theta}(r, \theta)=-\frac{1}{r \sin \theta} \frac{\partial \psi}{\partial r} \\
\widehat{v}_{r}(r, \theta)=\frac{1}{r^{2} \sin \theta} \frac{\partial \widehat{\psi}}{\partial \theta}, & \widehat{v}_{\theta}(r, \theta)=-\frac{1}{r \sin \theta} \frac{\partial \psi}{\partial r}
\end{array}
$$

The continuity equations outside and inside the liquid drop are automatically satisfied.

The perturbation parameter is the Reynolds number, Re, defined in terms of the parameters of the flow outside the drop by

$$
\operatorname{Re}=\frac{U a}{v}
$$

where $v=\eta / \rho$ is the kinematic viscosity and $\eta$ is the shear viscosity of the exterior fluid. Taylor and Acrivos [6] used the method of matched asymptotic expansions outside the liquid drop to obtain an expansion uniformly valid in $r$ for $a \leq r \leq \infty$. The inner expansion outside the drop is the straightforward expansion in powers of Re. This inner expansion is used to analyse the exterior flow close to the drop which includes the region of positive vorticity. The deformation of the drop at zero order in Re was found to be zero. Hence the boundary condition for the first order in Re solution was imposed at $r=1$. The inner expansion for the stream function outside the drop to first order in Re found by Taylor and Acrivos [6] can be written as

$$
\begin{aligned}
\psi(r, \theta)=\frac{1}{4}( & r-1) \sin ^{2} \theta \\
\times & {\left[\left(1+\frac{1}{8}\left(\frac{2+3 \kappa}{1+\kappa}\right) \operatorname{Re}\right)\right.} \\
& \times\left(2 r-\frac{\kappa}{1+\kappa}-\frac{\kappa}{1+\kappa} \frac{1}{r}\right) \\
& -\frac{\operatorname{Re}}{8}\left(\frac{2+3 \kappa}{1+\kappa}\right) \\
& \times\left(2 r-\frac{\kappa}{1+\kappa}-\frac{\kappa}{5(1+\kappa)^{2}} \frac{1}{r}\right. \\
& \left.\left.-\frac{\kappa(6+5 \kappa)}{5(1+\kappa)^{2}} \frac{1}{r^{2}}\right) \cos \theta\right] \\
+ & \left(\operatorname{Re}^{2}\right),
\end{aligned}
$$

as $\operatorname{Re} \rightarrow 0$, where $\kappa$ is the ratio of the shear viscosity of the drop to the shear viscosity of the exterior fluid:

$$
\kappa=\frac{\widehat{\eta}}{\eta} .
$$

Since shear viscosity is nonnegative, the range of values of $\kappa$ is $0 \leq \kappa \leq \infty$. For an inviscid gas bubble, $\kappa=0$, while for a solid sphere, $\kappa=\infty$. The stream function inside the liquid drop to first order in Re can be written as

$$
\begin{aligned}
\widehat{\psi}(r, \theta)= & -\frac{r^{2}\left(1-r^{2}\right) \sin ^{2} \theta}{4(1+\kappa)} \\
\times & {\left[1+\frac{\kappa(2+3 \kappa)}{40(1+\kappa)^{2}} \operatorname{Re}\right.} \\
& \left.+\operatorname{Re} \frac{(2+3 \kappa)(5+4 \kappa)}{40(1+\kappa)^{2}}(1-r \cos \theta)\right] \\
& +O\left(\operatorname{Re}^{2}\right),
\end{aligned}
$$

as $\operatorname{Re} \rightarrow 0$.

The deformation of the drop to first order in $\mathrm{Re}$ is proportional to the Weber number We and depends on the density ratio $\gamma$ (and on $\kappa$ ) where

$$
\mathrm{We}=\frac{\rho a U^{2}}{\sigma}, \quad \gamma=\frac{\hat{\rho}}{\rho},
$$


and $\sigma$ is the uniform interfacial tension. The boundary conditions for the order Re solution were imposed on the zero order surface of the drop, $r=1$, which is not deformed. The results therefore apply only for small Weber number.

\section{Attached Region of Positive Vorticity Exterior to the Liquid Drop}

Consider first the fluid flow exterior to the liquid drop.

Since the flow is axisymmetric, the vorticity $\boldsymbol{\omega}$ expressed in terms of the stream function $\psi(r, \theta)$ is

$$
\boldsymbol{\omega}=-\frac{1}{r \sin \theta} D^{2} \psi \mathbf{e}_{\phi}=\omega_{\phi} \mathbf{e}_{\phi}
$$

where

$$
D^{2}=\frac{\partial^{2}}{\partial r^{2}}+\frac{\sin \theta}{r^{2}} \frac{\partial}{\partial \theta}\left(\frac{1}{\sin \theta} \frac{\partial}{\partial \theta}\right)
$$

and $\mathbf{e}_{\phi}$ is the unit base vector parallel to the $\phi$-coordinate line in the direction of increase of $\phi$. For the exterior flow close to the drop the stream function is given by (3). With the aid of the identities

$$
\begin{aligned}
D^{2}\left(f(r) \sin ^{2} \theta\right) & =\left(\frac{d^{2} f}{d r^{2}}-\frac{2}{r^{2}} f\right) \sin ^{2} \theta, \\
D^{2}\left(f(r) \sin ^{2} \theta \cos \theta\right) & =\left(\frac{d^{2} f}{d r^{2}}-\frac{6}{r^{2}} f\right) \sin ^{2} \theta \cos \theta,
\end{aligned}
$$

it can be verified that

$$
\begin{aligned}
\omega_{\phi}(r, \theta)= & -\frac{1}{2}\left(\frac{2+3 \kappa}{1+\kappa}\right) \frac{\sin \theta}{r} \\
& \times\left[\left(1+\left(\frac{2+3 \kappa}{1+\kappa}\right) \frac{\operatorname{Re}}{8}\right) \frac{1}{r}\right. \\
& +\frac{\operatorname{Re}}{8}\left\{4-3\left(\frac{2+3 \kappa}{1+\kappa}\right) \frac{1}{r}\right. \\
& +\frac{3 \kappa(4+5 \kappa)}{5(1+\kappa)^{2} r^{2}} \\
& \left.\left.-\frac{2 \kappa}{(1+\kappa) r^{3}}\right\} \cos \theta\right] .
\end{aligned}
$$

The curves $\omega_{\phi}=$ constant in an axial plane $\phi=$ constant are the equivorticity lines [10].

To zero order in $\mathrm{Re}$

$$
\omega_{\phi}=-\frac{1}{2}\left(\frac{2+3 \kappa}{1+\kappa}\right) \frac{\sin \theta}{r^{2}}
$$

and hence $\omega_{\phi}<0$ except when $\theta=0$ and $\theta=\pi$ in which case $\omega_{\phi}=0$. When $\operatorname{Re}>0, \omega_{\phi}$ may not always be negative everywhere. We will show that a region of positive vorticity exists behind the drop and that it is attached to the surface of the drop. We will then investigate the properties of this region of positive vorticity.
From $(10), \omega_{\phi}(r, \theta)>0$ if $\cos \theta>H(r ; \kappa, \mathrm{Re})$, where

$$
\begin{aligned}
H(r ; \kappa, \mathrm{Re})= & \left(\frac{8}{\operatorname{Re}}+\frac{2+3 \kappa}{1+\kappa}\right) 5(1+\kappa)^{2} r^{2} \\
& \times\left(\left[-20(1+\kappa)^{2} r^{3}\right.\right. \\
& +15(1+\kappa)(2+3 \kappa) r^{2} \\
& \quad-3 \kappa(4+5 \kappa) r+10 \kappa(1+\kappa)])^{-1} .
\end{aligned}
$$

The dividing curve in an axial plane $\phi=$ constant between a region of positive vorticity and a region of negative vorticity outside the drop is

$$
\cos \theta=H(r ; \kappa, \mathrm{Re}) .
$$

The curve will be attached to the surface of the drop, $r=1$, if there is a solution, $\theta=\theta_{P}$, to the equation

$$
\begin{aligned}
\cos \theta_{P} & =H(1 ; \kappa, \mathrm{Re}) \\
& =\frac{5(1+\kappa)[8(1+\kappa)+(2+3 \kappa) \mathrm{Re}]}{(5+4 \kappa)(2+5 \kappa) \operatorname{Re}} .
\end{aligned}
$$

Since $\cos \theta_{P} \leq 1$, (14) has a solution for $\theta_{P}$ only if $\operatorname{Re} \geq \operatorname{Re}_{P}$, where

$$
\operatorname{Re}_{P}=\frac{40(1+\kappa)^{2}}{\kappa(8+5 \kappa)}
$$

We have

$$
\begin{aligned}
& \operatorname{Re}_{P}=8\left(1+\frac{2}{5 \kappa}+\frac{9}{25 \kappa^{2}}+O\left(\frac{1}{\kappa^{3}}\right)\right) \quad \text { as } \kappa \longrightarrow \infty, \\
& \operatorname{Re}_{P}=\frac{5}{\kappa}\left(1+\frac{11}{8} \kappa+\frac{9}{64} \kappa^{2}+O\left(\kappa^{3}\right)\right) \quad \text { as } \kappa \longrightarrow 0 .
\end{aligned}
$$

If $\mathrm{Re}<\mathrm{Re}_{P}$, there is no region of positive vorticity attached to the surface of the drop. It can be shown that $\mathrm{Re}_{P}$ is a decreasing function of $\kappa$.

For an inviscid gas bubble, $\kappa=0$ and $\mathrm{Re}_{P}=\infty$. This indicates that there is not a region of positive vorticity attached to an inviscid gas bubble. For an inviscid gas bubble there is also no downstream wake with standing eddies because no vorticity is generated upstream on the surface of an inviscid bubble [5].

For a solid sphere, $\kappa=\infty$ and $\operatorname{Re}_{P}=8$. At $\operatorname{Re}=8$ the perturbation solution of Proudman and Pearson $[5,8,9]$ predicts that an attached wake consisting of two standing eddies first appears downstream of the solid sphere. The standing eddies are due to the accumulation of vorticity generated upstream on the surface of the sphere. For a solid sphere, the attached region of positive vorticity and the attached wake first appear at the same value of the Reynolds number, $\operatorname{Re}=8$.

For $0<\kappa<\infty$ the standing eddy is detached from the surface of the liquid drop. It first appears in the flow downstream of the drop at Reynolds number $\operatorname{Re}_{A}$. A perturbation solution with perturbation parameter $1 / \kappa$ as $\kappa \rightarrow \infty$, and a 
numerical solution have been derived for $\operatorname{Re}_{A}$ [5]. It can be shown that $\operatorname{Re}_{A}>\mathrm{Re}^{*}$, where

$$
\operatorname{Re}^{*}=\frac{120(2+3 \kappa)(1+\kappa)^{2}}{\kappa(2+3 \kappa)(4+5 \kappa)}
$$

It is readily verified that $\mathrm{Re}_{P}<\mathrm{Re}^{*}$, and hence the attached region of positive vorticity appears outside the drop before the appearance of the standing eddy.

The value of $\kappa$ for given $\mathrm{Re}>8$ at which the region of positive vorticity first occurs as $\kappa$ is increased from zero is obtained by solving (14) with $\theta_{P}=0$ for $\kappa$. This gives the quadratic equation

$$
5(\operatorname{Re}-8) \kappa^{2}+8(\operatorname{Re}-10) \kappa-40=0 .
$$

For $\operatorname{Re}>8$, (18) has one real positive root

$$
\kappa_{P}=\frac{-8(\operatorname{Re}-10)+[32 \operatorname{Re}(2 \mathrm{Re}-15)]^{1 / 2}}{10(\operatorname{Re}-8)} .
$$

Consider now the range of $\theta_{P}$ on the outer surface of the drop for $0<\kappa \leq \infty$ and $\operatorname{Re}_{P} \leq \operatorname{Re} \leq \infty$. It can be shown from (14) that

$$
\frac{\partial \theta_{P}}{\partial \operatorname{Re}}>0, \quad \frac{\partial \theta_{p}}{\partial \kappa}>0,
$$

and hence $\theta_{P}$ is an increasing function of Re and $\kappa$. Also,

$$
\begin{gathered}
\lim _{\operatorname{Re} \rightarrow \infty} \theta_{P}=\cos ^{-1}\left[\frac{5(1+\kappa)(2+3 \kappa)}{(5+4 \kappa)(2+5 \kappa)}\right], \\
\lim _{\kappa \rightarrow \infty} \theta_{P}=\cos ^{-1}\left[\frac{3}{4}+\frac{2}{\mathrm{Re}}\right] .
\end{gathered}
$$

Equation (21) gives the maximum value of $\theta_{P}$ for a given value of $\kappa$ while (22) gives the maximum value of $\theta_{P}$ for a given value of Re. Further

$$
\lim _{\operatorname{Re} \rightarrow \infty} \lim _{\kappa \rightarrow \infty} \theta_{P}=\lim _{\kappa \rightarrow \infty} \lim _{\operatorname{Re} \rightarrow \infty} \theta_{P}=\cos ^{-1}\left(\frac{3}{4}\right)=41.4^{0} .
$$

For $\operatorname{Re}_{P} \leq \operatorname{Re} \leq \infty, \theta_{P}$ lies in the range $0 \leq \theta_{P} \leq 41.4^{0}$. When $\operatorname{Re}=\operatorname{Re}_{P}, \theta_{P}=0$ and when $\operatorname{Re}=\infty$ and $\kappa=\infty, \theta_{P}=$ $41.4^{0}$. In Figure $2, \theta_{P}$ is plotted against Re for a range of values of $\kappa$. Each curve starts at $\theta_{P}=0, \mathrm{Re}=\mathrm{Re}_{P}$, increases steadily for $\operatorname{Re}>\operatorname{Re}_{P}$, and tends to (21) for large values of Re. The distribution of vorticity over the outer surface of the drop, $w_{\phi}(1, \theta)$, is illustrated in Figures 3 and 4 . The vorticity is positive on the outer surface of the drop for $0 \leq \theta<\theta_{P}$ provided either $\operatorname{Re}>\operatorname{Re}_{P}$ or $\kappa>\kappa_{P}$.

Consider now the maximum point of extension, $r=r_{P}$, of the region of positive vorticity downstream of the drop. By putting $\theta=0$ in (13), we find that $r_{P}$ satisfies the cubic equation

$$
Q(r)=0
$$

where

$$
\begin{aligned}
Q(r)= & r^{3}-\left(\frac{2+3 \kappa}{2(1+\kappa)}-\frac{2}{\mathrm{Re}}\right) r^{2} \\
& +\frac{3 \kappa(4+5 \kappa)}{20(1+\kappa)^{2}} r-\frac{\kappa}{2(1+\kappa)}
\end{aligned}
$$

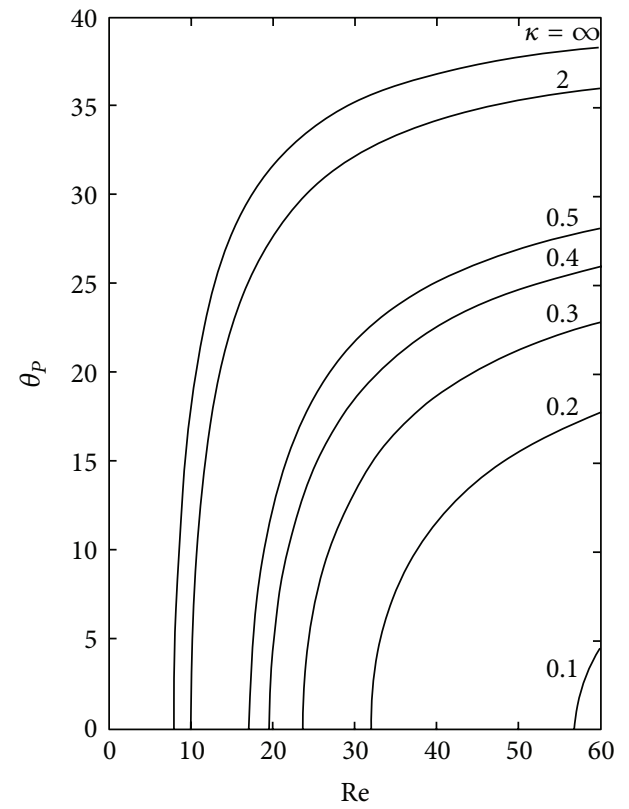

FIgURE 2: Graphs of $\theta_{P}$ plotted against Re for a range of values of $\kappa$.

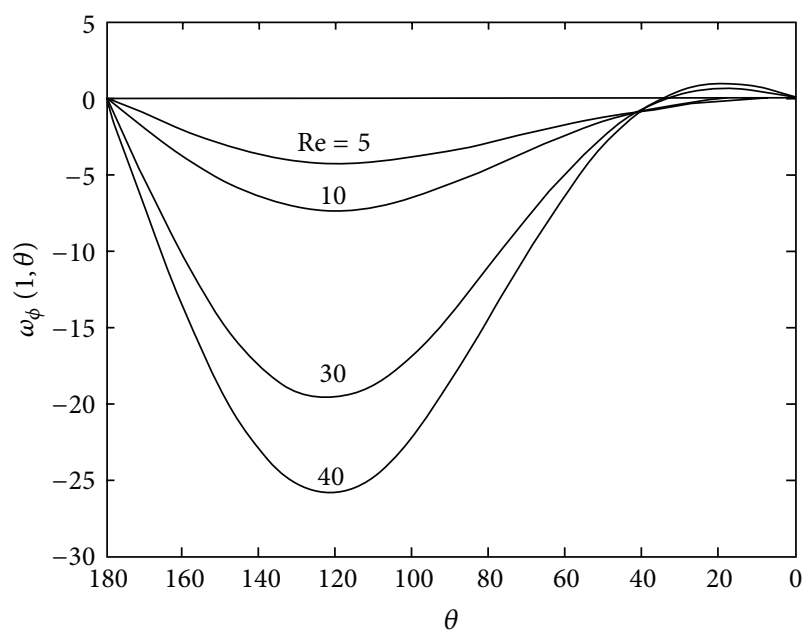

FIGURE 3: Graphs of $\omega_{\phi}(1, \theta)$ plotted against $\theta$ for $\kappa=2$ and a range of values of Re. When $\kappa=2, \operatorname{Re}_{P}=10$.

When $\kappa=0$, which describes an inviscid gas bubble, (24) reduces to

$$
r^{2}\left[r-\left(1-\frac{2}{\mathrm{Re}}\right)\right]=0 .
$$

The roots of (26) are $r=0$ (twice) and $r=1-2 /$ Re which do not lie outside the inviscid bubble. These results indicate that there is not a region of positive vorticity in the flow outside an inviscid gas bubble. We therefore consider $\kappa>0$.

Consider first $\operatorname{Re}=\operatorname{Re}_{P}$. Then (24) reduces to

$$
(r-1)\left[20(1+\kappa)^{2} r^{2}-\kappa(2+5 \kappa) r+10 \kappa(1+\kappa)\right]=0,
$$

which has one real root, $r=1$, and two complex conjugate roots. When $\mathrm{Re}=\mathrm{Re}_{P}, r_{P}=1$ and $\theta_{P}=0$. The region of positive vorticity first appears behind the drop. 


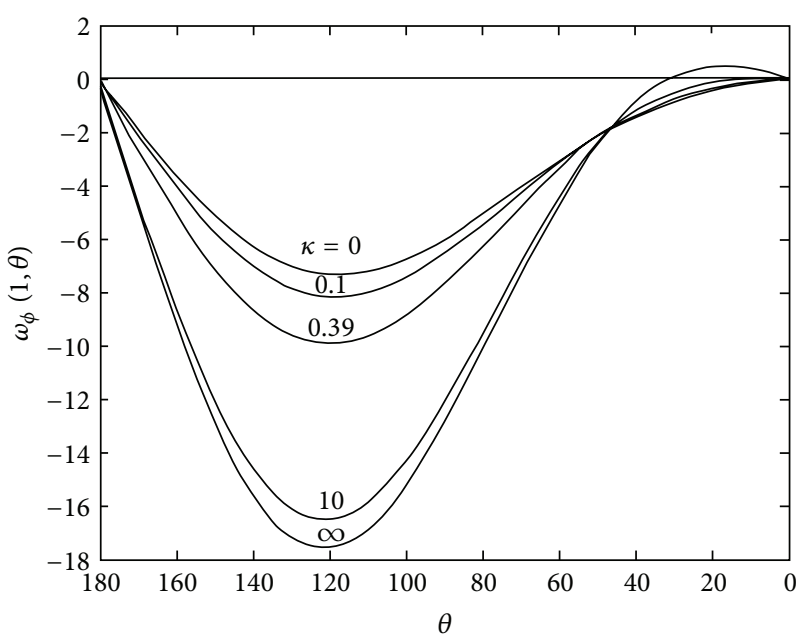

Figure 4: Graphs of $\omega_{\phi}(1, \theta)$ plotted against $\theta$ for $\operatorname{Re}=20$ and a range of values of $\kappa$. When $\operatorname{Re}=20, \kappa_{P}=0.39$.

Consider next $\operatorname{Re}>\operatorname{Re}_{P}$. We first show that (24) always has a positive real root greater than unity. Now

$$
Q(1)=-\frac{2\left(\operatorname{Re}-\operatorname{Re}_{P}\right)}{\operatorname{Re}^{2} e_{P}},
$$

and hence $Q(1)<0$ since $\operatorname{Re}>\operatorname{Re}_{P}$. Also since $Q(r) \rightarrow \infty$ as $r \rightarrow \infty$, it follows that $Q(r)=0$ for some $r=r_{P}>1$. This root is the end point on the axis of symmetry $\theta=0$ of the region of positive vorticity.

Since the production of vorticity at the interface increases as $\kappa$ and Re increase, we can expect that $r_{P}$ attains its maximum value when $\kappa=\infty$ and $\operatorname{Re}=\infty$. We have seen that this is indeed the case for $\theta_{P}$ which attains its maximum value when $\kappa=\infty$ and $\operatorname{Re}=\infty$. When $\kappa=\infty$ and $\operatorname{Re}=\infty$, (24) becomes

$$
r^{3}-\frac{3}{2} r^{2}+\frac{3}{4} r-\frac{1}{2}=0 .
$$

Now the standard form of a cubic equation is [11]

$$
s^{3}+3 H s+G=0 .
$$

In order to transform (29) to the standard form, let

$$
r=s+\frac{1}{2}
$$

Then (29) becomes

$$
s^{3}-\frac{3}{8}=0
$$

Thus $G^{2}+4 H^{3}>0$ and there is one real root and two complex conjugate roots [11]. The real root is $s=0.72$, and hence

$$
r_{P}(\max )=1.22 \text {. }
$$

The region of positive vorticity behind the drop is therefore a thin layer attached to the rear surface of the drop. Its

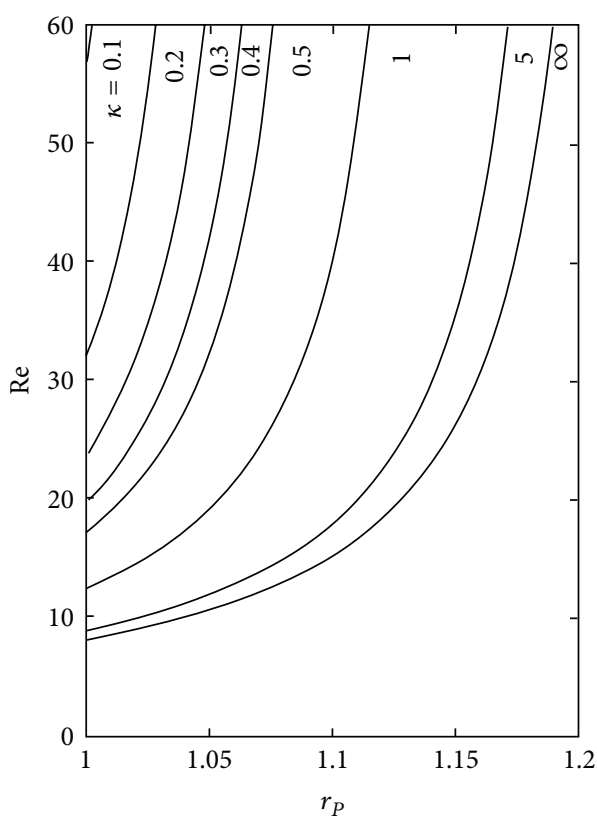

FIGURE 5: The numerical solution for $r_{p}$, the end point of the region of positive vorticity behind the drop, plotted against Re for a range of values of $\kappa$.

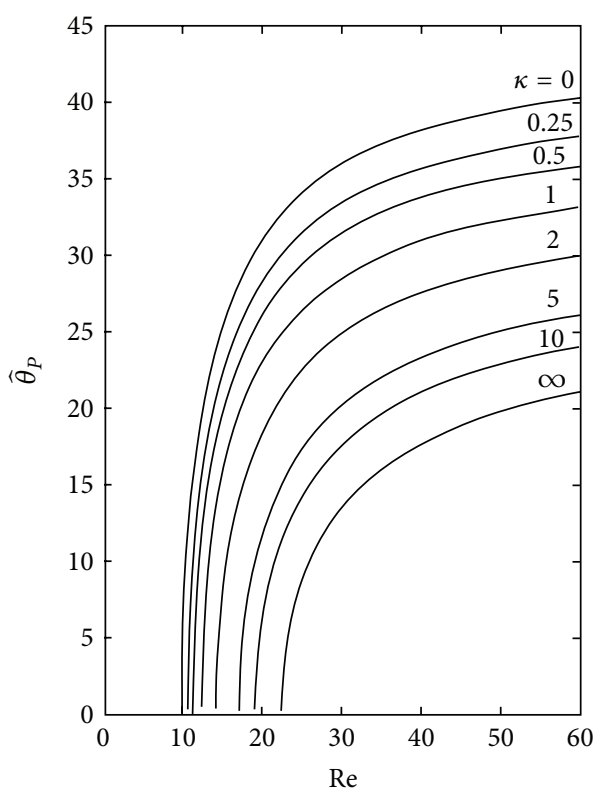

FIgURE 6: Graphs of $\widehat{\theta}_{P}$ plotted against Re for a range of values of $\kappa$.

maximum extension is $0 \leq \theta \leq 41.4^{0}$ and $1 \leq r \leq 1.22$. The dividing curve (13) generates a surface of revolution about the line $\theta=0$ which encloses the attached region of positive vorticity downstream of the drop.

A straightforward perturbation solution of (24) for $r_{P}$ in terms of the perturbation parameter

$$
\varepsilon=\frac{\operatorname{Re}-\operatorname{Re}_{P}}{\operatorname{Re}_{P}}, \quad \operatorname{Re}>\operatorname{Re}_{P},
$$




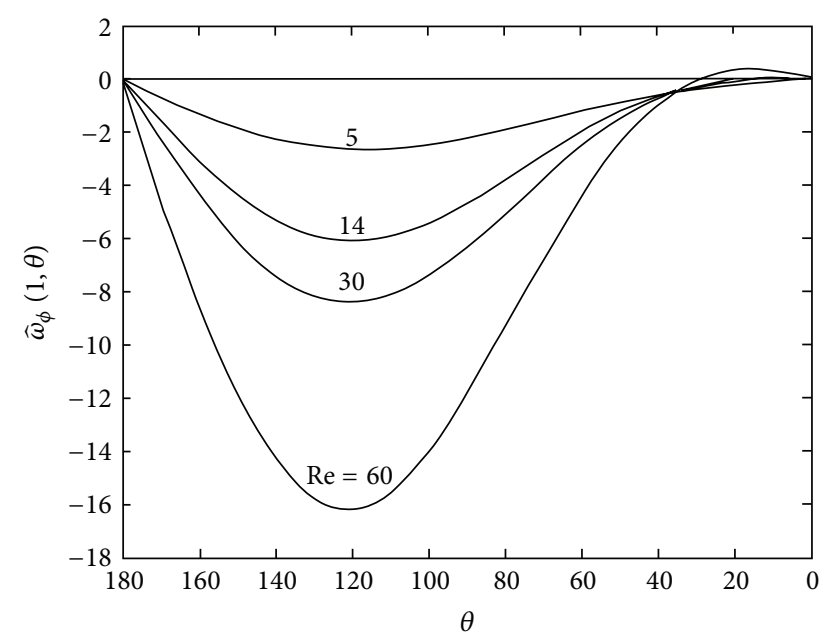

FIGURE 7: Graphs of $\widehat{\omega}_{\phi}(1, \theta)$ plotted against $\theta$ for $\kappa=2$ and a range of values of Re. When $\kappa=2, \widehat{\operatorname{Re}}_{P}=14$.

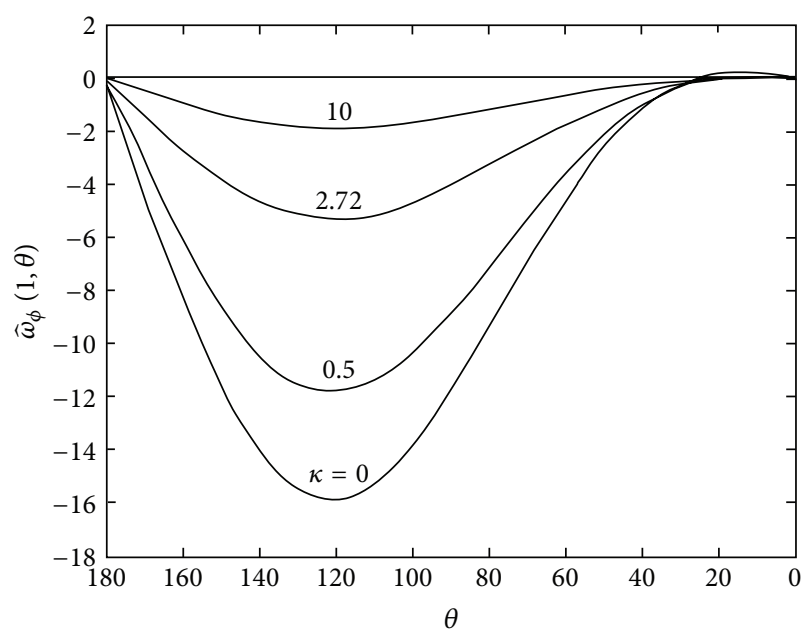

Figure 8: Graphs of $\widehat{\omega}_{\phi}(1, \theta)$ plotted against $\theta$ for $\mathrm{Re}=15$ and a range of values of $\kappa$. When $\operatorname{Re}=15, \widehat{\kappa}_{P}=2.72$.

is readily derived:

$$
r_{P}=1+\frac{\kappa(8+5 \kappa)}{\left(25 \kappa^{2}+48 \kappa+20\right)} \varepsilon+O\left(\varepsilon^{2}\right)
$$

as $\varepsilon \rightarrow 0$. For small $\kappa$,

$$
r_{P}-1=\frac{2 \kappa}{5}(1+O(\kappa)) \varepsilon, \quad \text { as } \kappa \longrightarrow 0,
$$

while for large $\kappa$,

$$
r_{P}-1=\frac{1}{5}\left(1-\frac{8}{25 \kappa}+O\left(\frac{1}{\kappa^{2}}\right)\right) \varepsilon, \quad \text { as } \kappa \longrightarrow \infty .
$$

Equations (36) and (37) give an approximation to the maximum thickness of the region of positive vorticity for small $\varepsilon$ when $\kappa$ is small and $\kappa$ is large, respectively.

The numerical solution for the real root of (24) is plotted in Figure 5. For each value of $\kappa$ the curve starts at $r=1, \operatorname{Re}=$
$\operatorname{Re}_{P}$. The graphs clearly show that $r_{P}$ increases as both $\kappa$ and Re increase and that (33) gives an upper bound for $r_{P}$.

We have seen that if $\operatorname{Re}$ or $\kappa$ are sufficiently large, a thin region of positive vorticity exists behind the drop and is attached to the surface. It appears before the appearance of the standing eddies and occurs due to the accumulation of positive vorticity at the outer surface of the drop. The size of the region of positive vorticity increases as each of $\mathrm{Re}$ and $\kappa$ increases because the generation of positive vorticity at the outer surface increases as Re and $\kappa$ increase as shown in Figures 3 and 4.

\section{Region of Positive Vorticity inside the Liquid Drop}

Inside the drop the equivorticity lines have more structure than the streamlines [5]. In this section we investigate the region of positive vorticity inside the drop and compare its properties with the properties of the attached region of positive vorticity outside the drop.

The vorticity $\widehat{\boldsymbol{\omega}}$ inside the drop is given by

$$
\widehat{\boldsymbol{\omega}}=-\frac{1}{r \sin \theta} D^{2} \widehat{\psi} \mathbf{e}_{\phi}=\widehat{\omega}_{\phi} \mathbf{e}_{\phi},
$$

where $D^{2}$ and $\widehat{\psi}(r, \theta)$ are defined by (8) and (5). With the aid of the identities (9) it can be shown that

$$
\begin{aligned}
\widehat{\omega}_{\phi}(r, \theta)= & -\frac{5 r \sin \theta}{2(1+\kappa)} \\
\times & {\left[1+\frac{\operatorname{Re}}{8}\left(\frac{2+3 \kappa}{1+\kappa}\right)\right.} \\
& \left.\quad 7 \operatorname{Re} \frac{(2+3 \kappa)(5+4 \kappa)}{200(1+\kappa)^{2}} r \cos \theta\right] .
\end{aligned}
$$

To zero order in Re,

$$
\widehat{\omega}_{\phi}(r, \theta)=-\frac{5 r \sin \theta}{2(1+\kappa)} .
$$

Thus when $\operatorname{Re}=0, \widehat{\omega}_{\phi}(r, \theta)<0$ if $\theta \neq 0$ and $\theta \neq \pi$. The equivorticity lines, $\omega_{\phi}=$ constant, in the plane $\phi=$ constant are straight lines parallel to the axis of symmetry, $\theta=0$.

Consider now $\widehat{\omega}_{\phi}(r, \theta)$ to first order in Re. From (39), $\widehat{\omega}_{\phi}(r, \theta)>0$ if

$$
r \cos \theta>\frac{25(1+\kappa)[8(1+\kappa)+\operatorname{Re}(2+3 \kappa)]}{7(2+3 \kappa)(5+4 \kappa) \operatorname{Re}} .
$$

The region of positive vorticity therefore consists of the part of the drop

$$
\frac{25(1+\kappa)[8(1+\kappa)+\operatorname{Re}(2+3 \kappa)]}{7(2+3 \kappa)(5+4 \kappa) \operatorname{Re}}<r \cos \theta<1 .
$$

It exists provided $\operatorname{Re}>\widehat{\operatorname{Re}}_{P}$, where

$$
\widehat{\operatorname{Re}}_{P}=\frac{200(1+\kappa)^{2}}{(2+3 \kappa)(10+3 \kappa)} .
$$




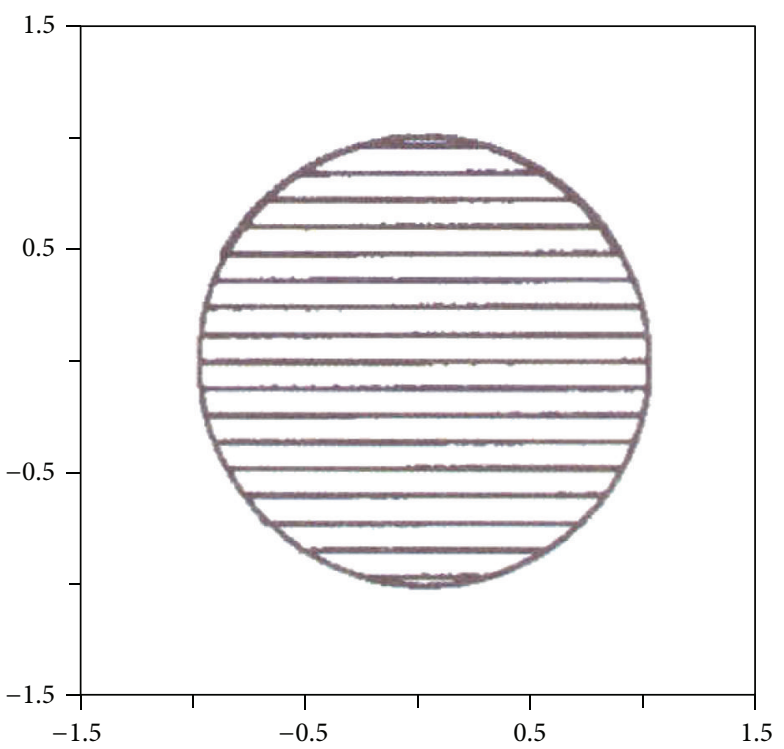

(a) $\mathrm{Re}=0$

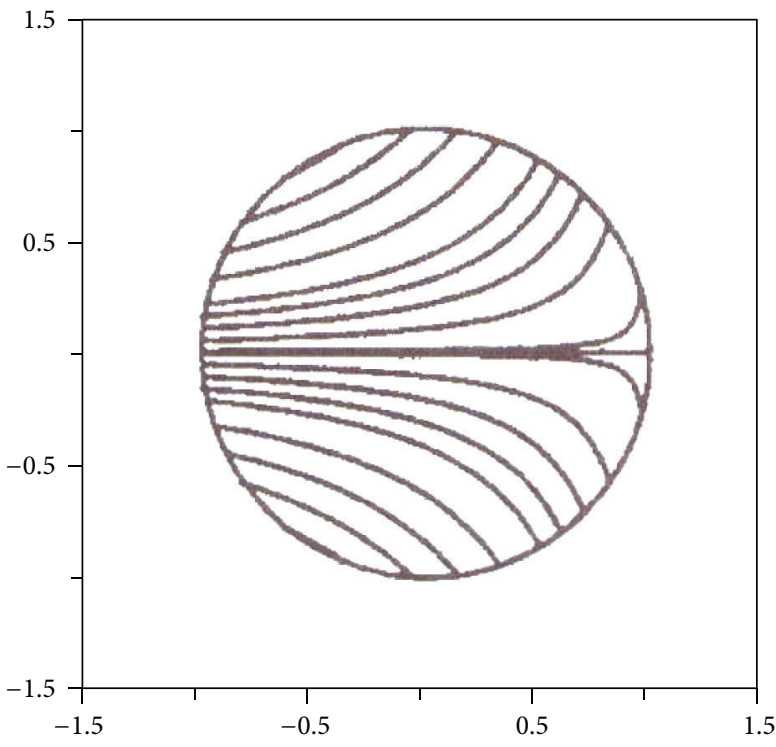

(c) $\operatorname{Re}=14$

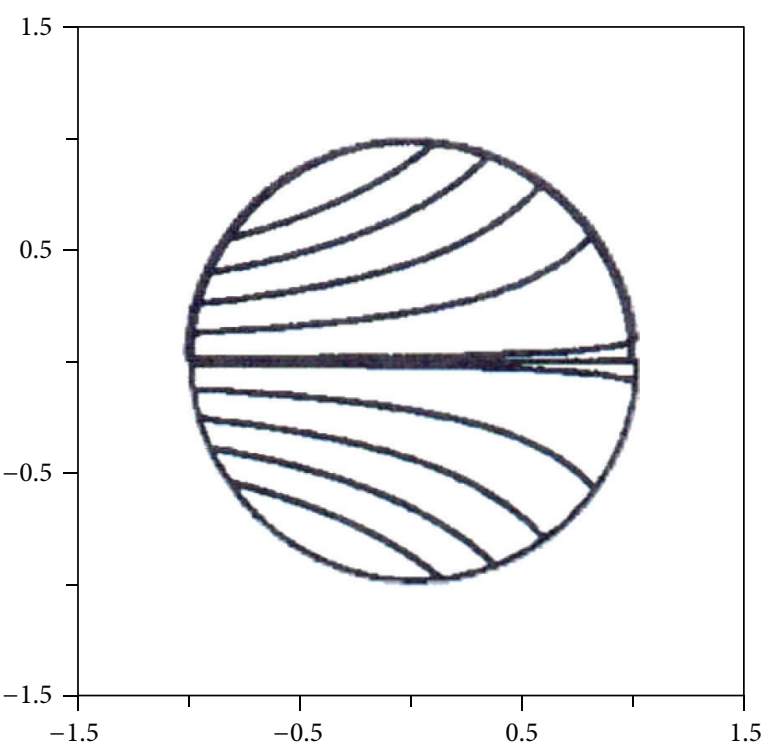

(b) $\operatorname{Re}=5$

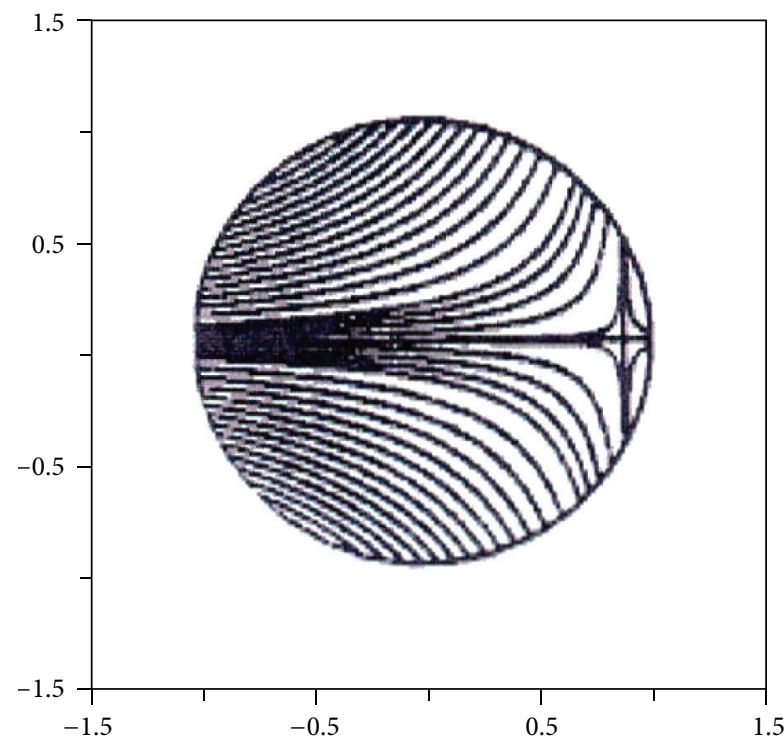

(d) $\operatorname{Re}=40$

FIGURE 9: Equivorticity lines inside the drop in an axial plane for $\kappa=2$ and $\operatorname{Re}=0,5,14$, and 40 . When $\kappa=2, \widehat{\operatorname{Re}}_{P}=14$.

We have

$$
\begin{aligned}
& \widehat{\operatorname{Re}}_{P}=\frac{200}{9}\left[1-\frac{2}{\kappa}+\frac{61}{9 \kappa^{2}}+O\left(\frac{1}{\kappa^{3}}\right)\right] \quad \text { as } \kappa \longrightarrow \infty \\
& \widehat{\operatorname{Re}}_{P}=10\left[1+\frac{\kappa}{5}+\frac{19}{100} \kappa^{2}+O\left(\frac{1}{\kappa^{3}}\right)\right] \quad \text { as } \kappa \longrightarrow 0
\end{aligned}
$$

It can be shown that $\widehat{\operatorname{Re}}_{P}$ is an increasing function of $\kappa$, which compares with $\operatorname{Re}_{P}$ which is a decreasing function of $\kappa$. The Reynolds number $\widehat{\operatorname{Re}}_{P}$ increases steadily from 10 at $\kappa=0$ for an inviscid gas bubble to 22.22 at $\kappa=\infty$ for a solid sphere. For $\operatorname{Re}<10$, the region of positive vorticity inside the drop does not exist for any value of $\kappa$ while for Re $>22.22$ it exists for all values of $\kappa$.
The value of $\kappa$ for given Re at which the region of positive vorticity first occurs as $\kappa$ decreases from $\kappa=\infty$ is obtained by solving (43) for $\kappa$. This yields the quadratic equation

$$
(200-9 \mathrm{Re}) \kappa^{2}+4(100-9 \mathrm{Re}) \kappa+20(10-\mathrm{Re})=0 .
$$

For $10<\operatorname{Re}<22.22$, (45) has one real positive root:

$$
\widehat{\kappa}_{P}=\frac{-2(100-9 \mathrm{Re})+2[2 \operatorname{Re}(18 \mathrm{Re}-175)]^{1 / 2}}{(200-9 \mathrm{Re})} .
$$

The region of positive vorticity exists for $0 \leq \kappa<\widehat{\kappa}_{P}$. It does not exist for $\kappa>\widehat{\kappa}_{P}$. 


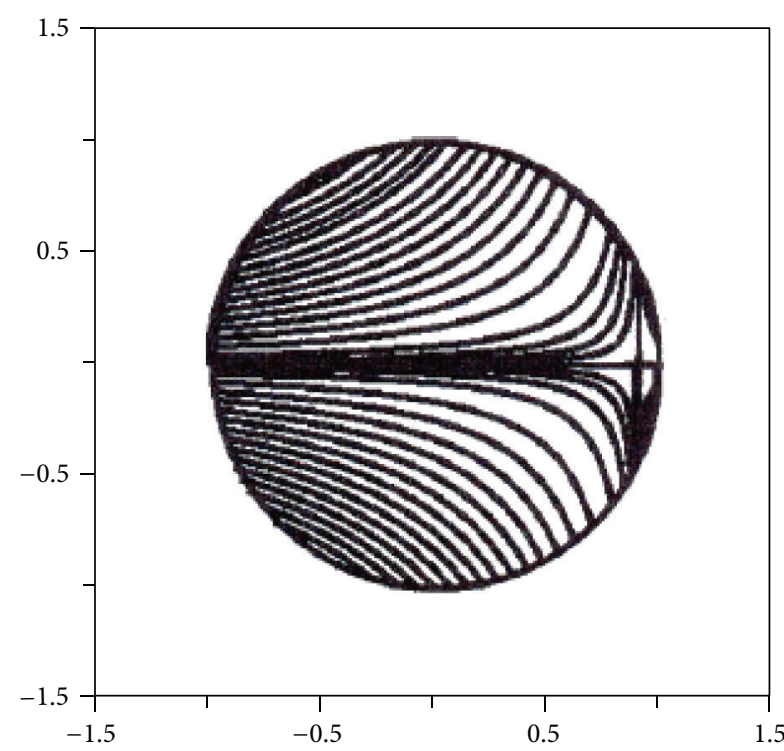

(a) $\kappa=0$

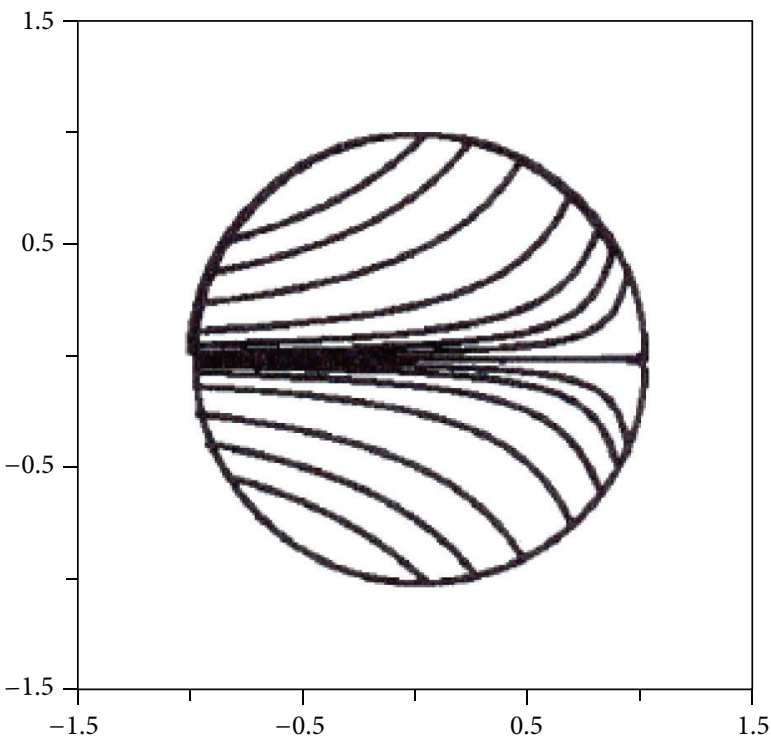

(c) $\kappa=2.72$

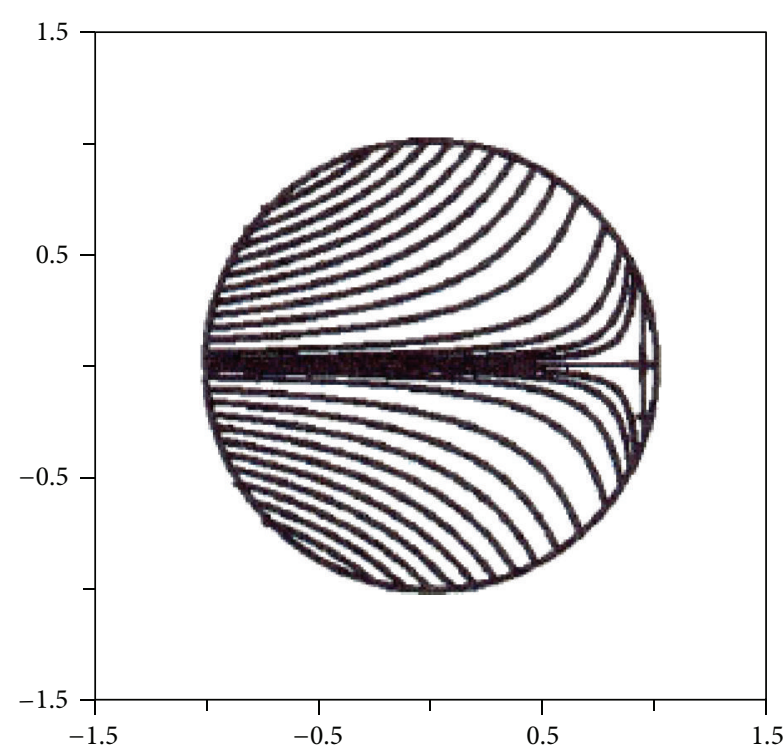

(b) $\kappa=0.5$

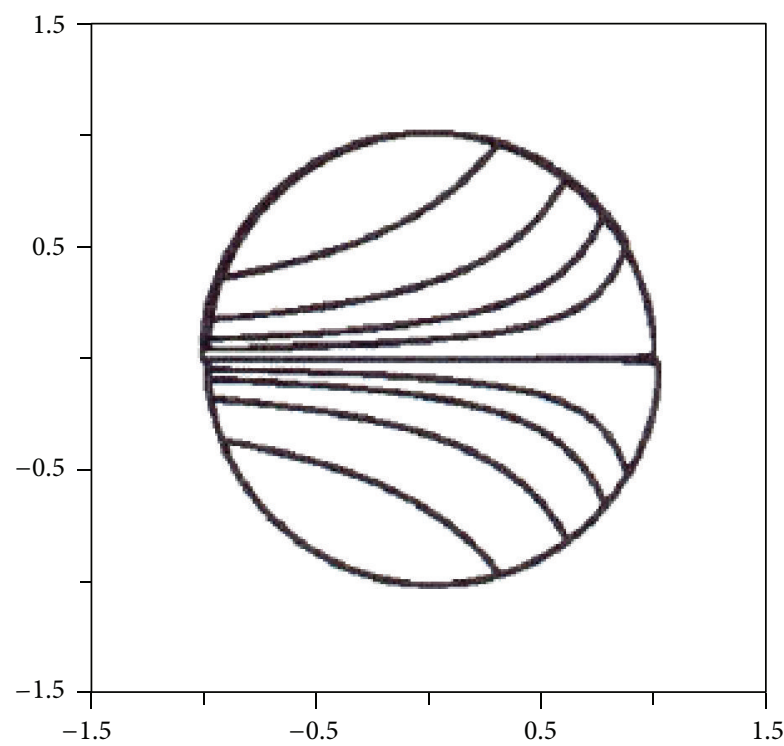

(d) $\kappa=10$

FIGURE 10: Equivorticity lines inside the drop in an axial plane for $\operatorname{Re}=15$ and $\kappa=0,0.5,2.72$, and 10. When $\operatorname{Re}=15, \widehat{\kappa}_{P}=2.72$.

On the inner surface of the liquid drop the region of positive vorticity extends over the range $0 \leq \theta \leq \widehat{\theta}_{P}$, where $\widehat{\theta}_{P}$ is obtained from (41) with $r=1$ :

$$
\widehat{\theta}_{P}=\cos ^{-1}\left[\frac{25(1+\kappa)[8(1+\kappa)+(2+3 \kappa) \operatorname{Re}]}{7(2+3 \kappa)(5+4 \kappa) \operatorname{Re}}\right] .
$$

It can be verified that

$$
\frac{\partial \widehat{\theta}_{P}}{\partial \operatorname{Re}}>0, \quad \frac{\partial \hat{\theta}_{P}}{\partial \kappa}<0,
$$

and hence $\widehat{\theta}_{P}$ is an increasing function of Re and a decreasing function of $\kappa$. Also

$$
\begin{aligned}
& \lim _{\operatorname{Re} \rightarrow \infty} \widehat{\theta}_{P}=\cos ^{-1}\left[\frac{25(1+\kappa)}{7(5+4 \kappa)}\right], \\
& \lim _{\kappa \rightarrow 0} \widehat{\theta}_{P}=\cos ^{-1}\left[\frac{5}{7}\left(1+\frac{4}{\operatorname{Re}}\right)\right] .
\end{aligned}
$$

Equation (49) gives the maximum value of $\widehat{\theta}_{P}$ for a given value of $\kappa$ while (50) gives the maximum value of $\widehat{\theta}_{P}$ for a given value of Re. Further,

$$
\lim _{\operatorname{Re} \rightarrow \infty} \lim _{\kappa \rightarrow 0} \hat{\theta}_{P}=\lim _{\kappa \rightarrow 0} \lim _{\operatorname{Re} \rightarrow \infty} \hat{\theta}_{P}=\cos ^{-1}\left(\frac{5}{7}\right)=44.4^{0} .
$$




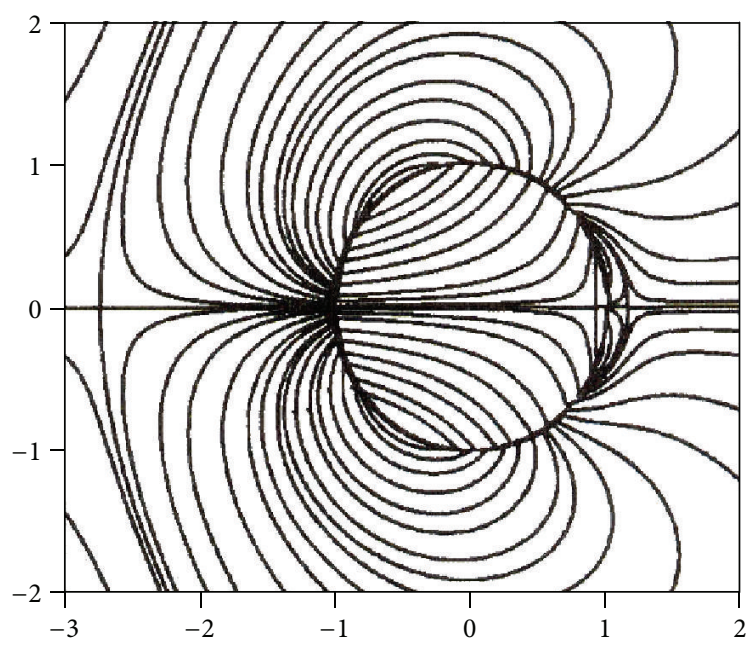

FIGURE 11: Equivorticity lines for flow past a drop with $\kappa=5$ and $\operatorname{Re}=40$. The direction of flow is from left to right.

Equation (51) gives the maximum value of $\widehat{\theta}_{P}$. It compares with $41.4^{0}$ for the maximum value of $\theta_{P}$ derived in (23). In Figure $6, \widehat{\theta}_{P}$ is plotted against Re for values of $\kappa$ ranging from 0 to $\infty$. The curves are confined to a narrow band emanating from $10 \leq \operatorname{Re} \leq 22.22$. The limiting cases, $\kappa=\infty$ and $\kappa=0$, are reversed in Figures 2 and 6 . The distribution of vorticity over the inner surface of the drop, $\widehat{\omega}_{\phi}(1, \theta)$, is shown in Figures 7 and 8 . The vorticity is positive on the inner surface for $0<\theta<\widehat{\theta}_{P}$ provided $\operatorname{Re}>\widehat{\operatorname{Re}}_{P}$ or $\kappa<\widehat{\kappa}_{P}$. The vorticity is discontinuous across the interface $r=1$ and the angles $\theta_{P}$ and $\widehat{\theta}_{P}$ are different for given values of $\kappa$ and Re.

In Figure 9 the equivorticity lines inside the drop are plotted for $\kappa=2$ and a range of values of Re. When $\kappa=2$, the region of positive vorticity exists for $\mathrm{Re}>\widehat{\mathrm{Re}}=14$. In Figure 10 the equivorticity lines inside the drop are plotted for $\operatorname{Re}=15$ and a range of values of $\kappa$. When $\operatorname{Re}=15$, the region of positive vorticity exists for $0 \leq \kappa<\widehat{\kappa}_{P}=2.72$.

If $0 \leq \kappa<1$, then $\widehat{\operatorname{Re}}_{P}<\operatorname{Re}_{P}$, and as $\mathrm{Re}$ increases from zero, the region of positive vorticity occurs inside the drop before it occurs outside and $\widehat{\theta}_{P}>\theta_{P}$. If $\kappa>1$, then $\mathrm{Re}_{P}<\widehat{\operatorname{Re}}_{P}$ and the region of positive vorticity occurs outside the drop before it occurs inside and $\theta_{P}>\widehat{\theta}_{P}$. When $\kappa<1$ the generation of vorticity at the interface due to the no-slip condition is more effective in the interior because the interior fluid is less viscous than the exterior fluid. When $\kappa>1$, the opposite is the case.

In Figure 11, the equivorticity lines inside and outside the drop are plotted for $\kappa=5$ and $\operatorname{Re}=40$. For these values of $\kappa$ and $\operatorname{Re}, \theta_{P}=36^{\circ}$ and $\hat{\theta}_{P}=23.4^{0}$. We see that there is also a region of positive vorticity upstream of the drop. In a diagram showing the streamlines there would be a standing eddy downstream of the drop since when $\kappa=5$ and $\operatorname{Re}=40$, $\operatorname{Re}>\operatorname{Re}_{A}[5]$.

\section{Concluding Remarks}

The significant fluid dynamical features in flow past a liquid drop are the detached wake behind the drop, the attached region of positive vorticity outside the drop, and the region of positive vorticity at the rear inside the drop. Using the perturbation solution of Taylor and Acrivos [6] to first order in $\mathrm{Re}$, we have derived in this paper analytical expressions for the main properties of the regions of positive vorticity outside and inside the drop. The results should be useful in numerical and experimental investigations when the Weber number, and therefore the deformation of the drop, is small. The analytical results are in qualitative agreement with the numerical results of Dandy and Leal [4] who present plots of the equivorticity lines and regions of positive vorticity in flow past a liquid drop. The expansions for large values of the viscosity ratio $\kappa$ should be useful in flow past a very viscous drop because they tend to results for a solid sphere derived from the perturbation solution of Proudman and Pearson [8] which are in good agreement with numerical and experimental results. The expansions for small values of $\kappa$ should be useful in flow past a gas bubble.

\section{Acknowledgment}

D. P. Mason thanks the National Research Foundation, Pretoria, South Africa, for the financial support.

\section{References}

[1] V. Y. Rwkind and G. Ryskin, "Flow structure in motion of a spherical drop in a fluid medium at intermediate Reynolds numbers," Fluid dynamics, vol. 11, pp. 5-12, 1976.

[2] D. L. R. Oliver and J. N. Chung, "Steady flow inside and around a fluid sphere at low Reynolds numbers," Journal of Fluid Mechanics, vol. 154, pp. 215-230, 1985.

[3] D. L. R. Oliver and J. N. Chung, "Flow about a fluid sphere at low to moderate Reynolds numbers," Journal of Fluid Mechanics, vol. 177, pp. 1-18, 1987.

[4] D. S. Dandy and L. G. Leal, "Buoyancy-driven motion of a deformable drop through a quiescent liquid at intermediate Reynolds number," Journal of Fluid Mechanics, vol. 208, pp. 161192, 1989.

[5] G. M. Moremedi and D. P. Mason, "Streamlines and detached wake in steady flow past a spherical liquid drop," Mathematical and Computational Applications, vol. 15, no. 4, pp. 543-557, 2010.

[6] T. D. Taylor and A. Acrivos, "On the deformation and drag of a falling viscous drop at low Reynolds number," Journal of Fluid Mechanics, vol. 18, pp. 466-476, 1964.

[7] R. M. Wellek, A. K. Agrawal, and A. H. P. Skelland, "Shape of liquid drops moving in liquid media," AIChE Journal, vol. 12, pp. 854-862, 1965.

[8] I. Proudman and J. R. A. Pearson, "Expansions at small Reynolds numbers for the flow past a sphere and a circular cylinder," Journal of Fluid Mechanics, vol. 2, pp. 237-262, 1957.

[9] M. Van Dyke, Perturbation Methods in Fluid Mechanics, Applied Mathematics and Mechanics, Academic Press, New York, NY, USA, 1964.

[10] S. C. R. Dennis and J. D. A. Walker, "Calculation of the steady flow past a sphere at low to moderate Reynolds numbers," Journal of Fluid Mechanics, vol. 48, pp. 771-789, 1971.

[11] W. Briggs and G. H. Bryan, The Tutorial Algebra, University Tutorial Press, London, UK, 1942. 


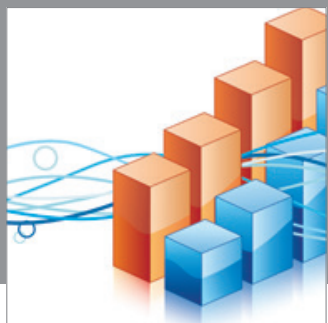

Advances in

Operations Research

mansans

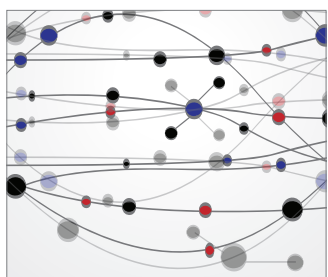

The Scientific World Journal
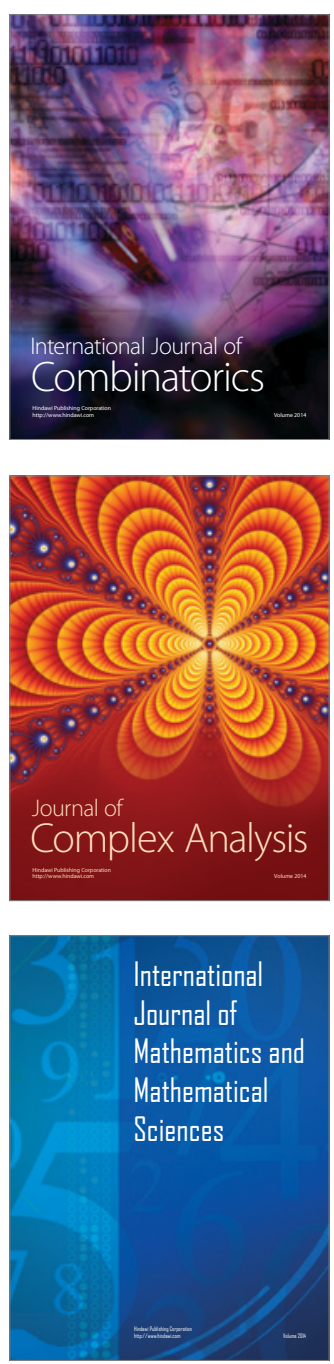
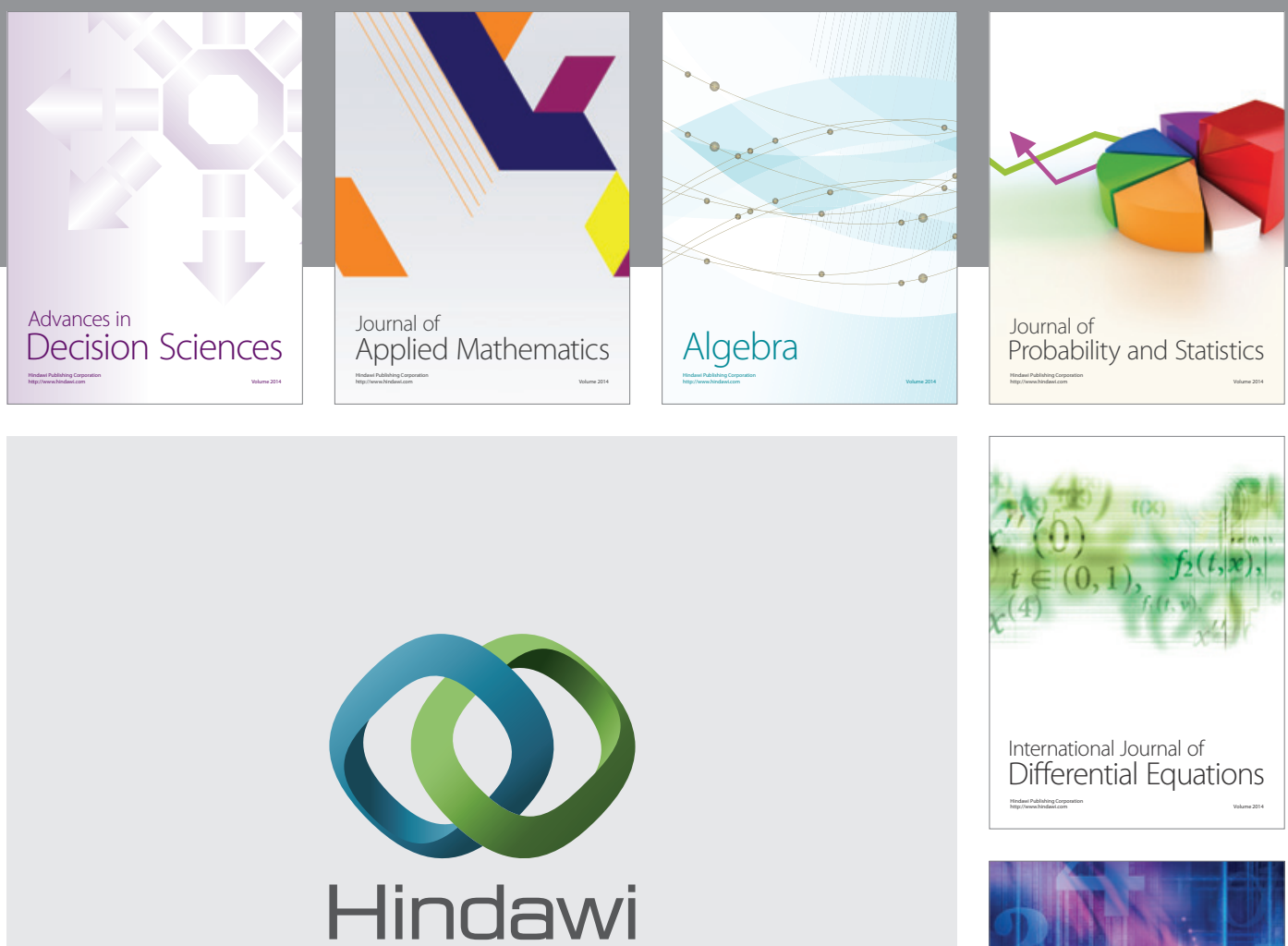

Submit your manuscripts at http://www.hindawi.com
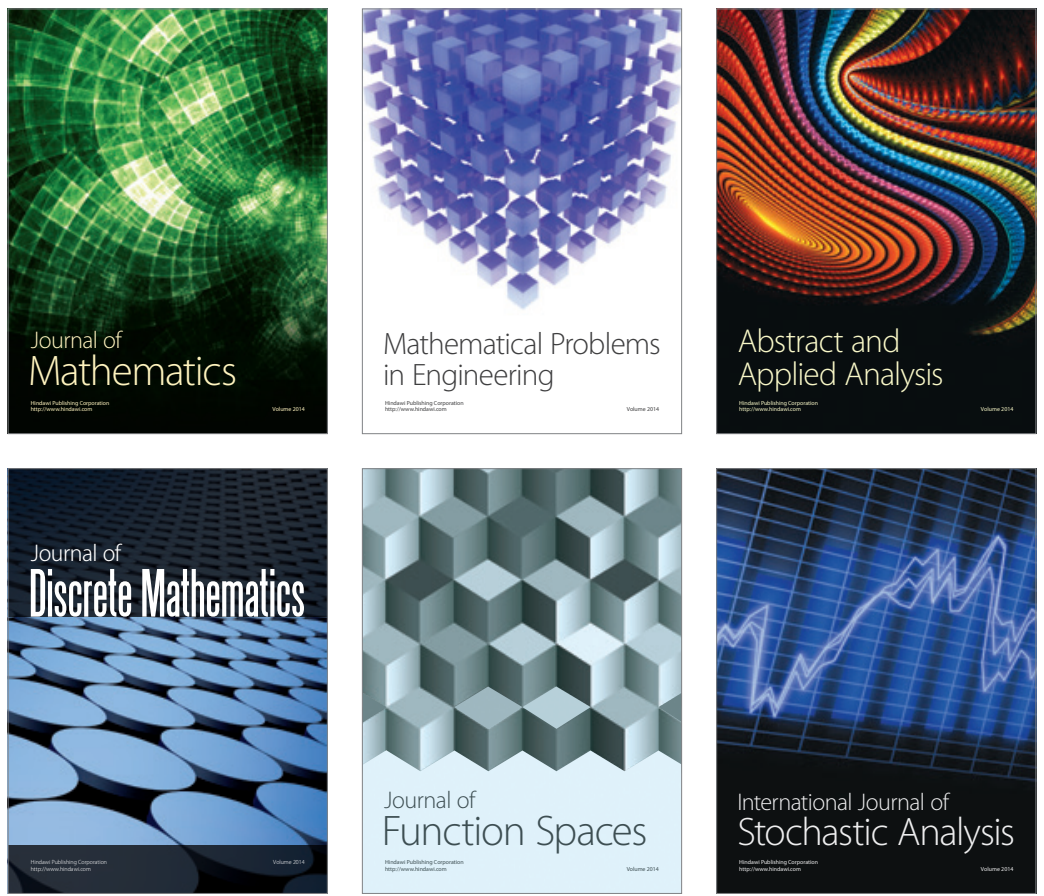

Journal of

Function Spaces

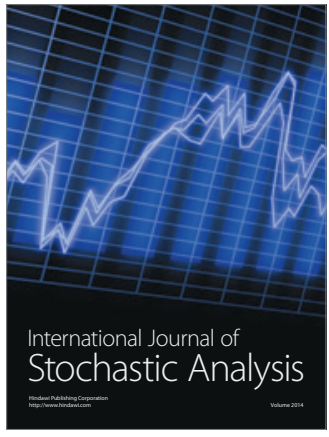

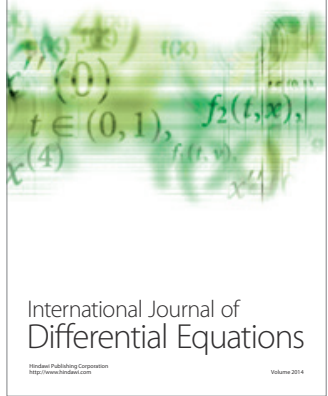
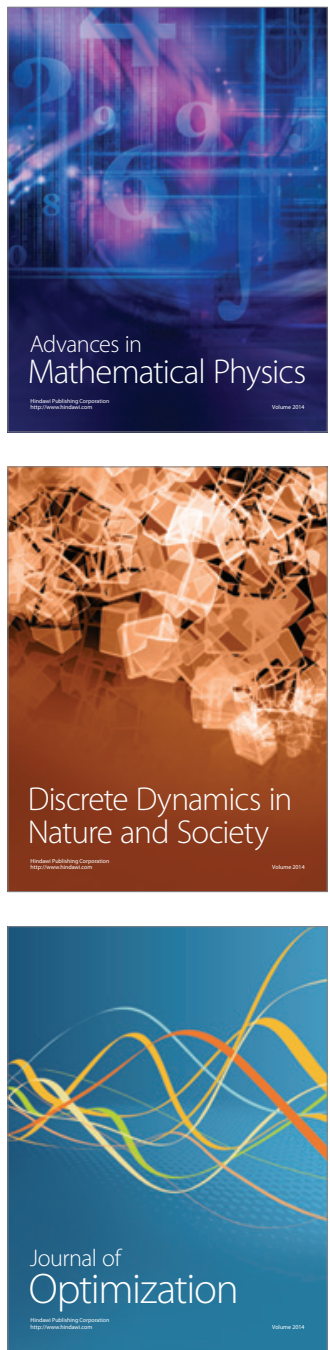